\title{
Optimal Replacement Age and Maintenance Cost: A Case Study
}

\author{
Nayeema Sultana, Md. RezaulKarim \\ Department of Statistics, University of Rajshahi, Rajshahi, Bangladesh
}

Email address:

nayeemaru@yahoo.com (N. Sultana),mrezakarim@yahoo.com (Md. R. Karim)

To cite this article:

Nayeema Sultana, Md. RezaulKarim. Optimal Replacement Age and Maintenance Cost: A Case Study. American Journal of Theoretical and Applied Statistics. Vol. 4, No. 2, 2015, pp. 53-57. doi: 10.11648/j.ajtas.20150402.12

\begin{abstract}
Maintenance plays a very vital role throughout an equipment/system's planned life-cycle. Maintenance costs contribute a major portion of the life cycle costs of an equipment or system. This paper analyzes a set of failure data of a particular type of battery that used in automobile and/or trailer and proposes the optimal maintenance age at which the nonfailed battery be maintained. A 2-fold Weibull mixture distribution is selected as the suitable distribution for the lifetime of the battery. The Expectation-Maximization (EM) algorithm is applied to estimate the parameters of the mixture distribution. The research will be of interest for effective maintenance management to maintenance engineers and managers working in battery manufacturing industry, as well as customers and service providers.
\end{abstract}

Keywords: Maintenance, 2-fold WeibullMixture Distribution, EM Algorithm, Optimal Replacement Age

\section{Introduction}

Regular maintenance is essential to keep equipment, machines and the work environment safe and reliable. It helps to eliminate workplace hazards. If maintenance is not carried out soon enough, is incorrectly carried out, or communications between maintenance and operation staff are not effective, the plant may fail dangerously during start up or during normal operation phase (Hale et al.,1998). Lack of maintenance or inadequate maintenance can lead to dangerous situations, accidents and health problems. Nowadays organizations are not only satisfied with keep machines in good conditions further, maintenance actions could be planned and totallyefficient. Maintenance sometimes could be a critical aspect in an organization and it could turn unnecessary cost into profits. In united states over than $\$ 600$ billion has been spent on critical plant systems although it has been increased to $\$ 800$ billion by 10 years (Mobley, 2004).

Economic impact of maintenance has gained importance and is still getting stronger. Eichler (1990) mentioned that 10 to $30 \%$ of all employees work in the maintenance sector; in highly automated industries over $60 \%$. This amount will increase due to higher automated production systems and, therefore, less operationally working personal.

The outline of the paper is as follows. Section 2 describes the basic concept of maintenance. Section 3 presents the mixture distributions. Detail analysis of the example data set (a case study) is given in Section 4. Section 5 concludes the paper.

\section{Maintenance}

The British Standards Institution defines maintenance as: "A combination of all technical and associated administrative activities required to keep an equipment, installations and other physical assets in the desired operating condition or restore them to this condition". The definition of maintenance may combine the technical and administrative actions that intended to retain an item or restore it to a state in which it can perform a required function. Maintenance provides crucial support for heavy and capital-intensive industry by keeping machinery and equipment in a safe operating condition. Therefore, maintenance plays a main role in sustaining long-term profitability and competitiveness for an organization (Aditya and Uday, 2006).

Two most commonly used maintenance policies are:

1. Optimal age-based preventive replacement (Type-I Policy)

2. Optimal constant-interval preventive replacement (Type-II Policy)

This paper considers the optimal age-based preventive replacement (Type-I Policy). A type I policy is defined 
as:perform preventive replacement after $t_{p}$ lifetimes (e.g., hours, days, moths, etc.) of continuing operation without failure; $t_{p}$ could be finite or infinite. In case of infinite $t_{p}$, no preventive maintenance (replacement) is scheduled. If the system fails prior to $t_{p}$ hours having elapsed, perform

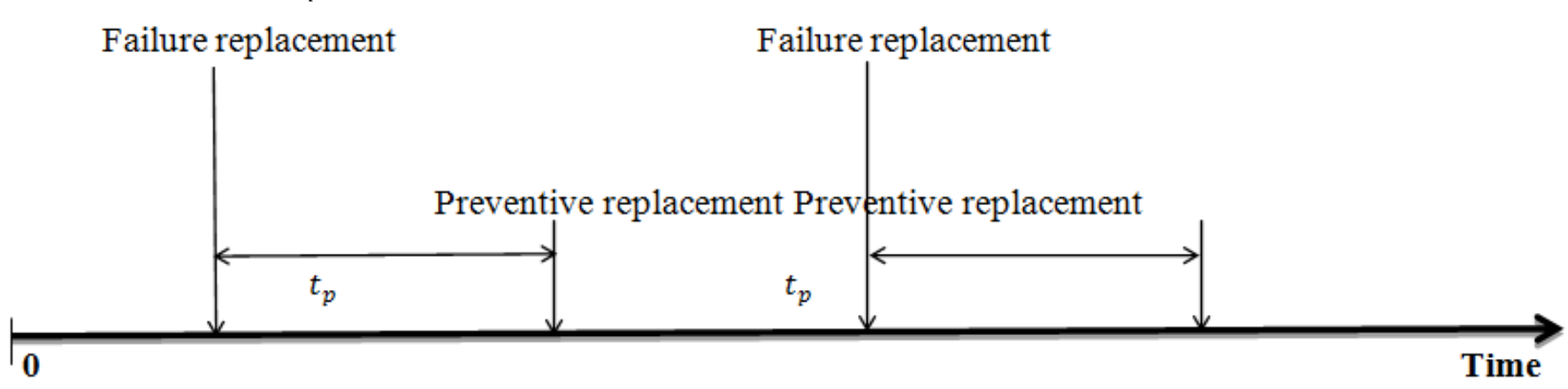

maintenance at the time of failure and reschedule thepreventive maintenance after $t_{p}$ operation times. In this policy, it is assume that the system is as good as new after preventive maintenance is performed. This policy illustrated in the Fig. 1.

Figure 1. Type-I policy cycles

Source: Duffuaa el at. (1999)

The objective function is the asymptotic expected cost per unit time. The asymptotic expected cost per unit time can be obtained as the ratio of the expected cycle cost (ECC) and the expected cycle length (ECL) as,

$$
J(T)=\frac{\text { Total expected cost per cycle (ECC) }}{\text { Expected cycle length (ECL) }}
$$

The time to failure for a battery, $X$, is a random variable with distribution function $F(x)$. A PM action results if $X \geq T$ in which case the cycle length is $T$ with probability $\bar{F}(t)$, (also known as reliability function of $T$ ) A CM action results when $X<T$ and the cycle length is $X$. As a result ECL is given by

$$
E C L=\int_{0}^{T} t f(t)+T \bar{F}(T)=\int_{0}^{T} \bar{F}(t) d t(1)
$$

Let $C_{f}$ and $C_{p}$ denote the average cost of a CM and PM replacement, respectively. As a result ECC is given by

$$
E C C=C_{f} F(T)+C_{p} \bar{F}(T)(2)
$$

From (1) and (2), the asymptotic average cost per unit time can be expressedas,

$$
J(T)=\frac{C_{f} F(T)+C_{p} \bar{F}(T)}{\int_{0}^{T} t f(t)+T \bar{F}(T)}
$$

This objective function will be used in this paper to determine the optimum maintenance age with respect to cost for the battery.

\section{Mixture Distribution}

A mixture distribution is a probabilistic distribution for representing the presence of subpopulations within an overall population, without requiring that an observed data set should identify the sub-population to which an individual observation belongs. Problems associated with "mixture distributions" relate to deriving the properties of the overall population from which these sub-populationsare taken.Mixture distributions are used to make statistical inferences about the properties of the sub-populations given only observations on the pooled population, without subpopulations identity information.

A general $K$-fold mixture model is given by the distribution function,

$$
F(t ; \theta)=\sum_{k=1}^{K} p_{k} F_{k}\left(t ; \theta_{k}\right)
$$

where $p_{k}$ are a set of probabilities and $F_{k}(\cdot)$ are the CDFs of the $k$ th sub-populationwith parameter $\theta_{k}, k=1,2, \ldots, K$, and the parameters $p_{k}$ are the mixing probability of the $k$ th subpopulation.Here, $p_{k} \geq 0, p_{1}+\cdots+p_{K}=1$.

For example, the mixture distributions can be used to model the variability resulting from parts being bought from $K$ different suppliers with $F_{k}(t)$ denoting the failure distribution for parts obtained from supplier, $k=1,2, \ldots, K$.

The Expectation-Maximization (EM) algorithmis an efficient iterative procedure to compute the Maximum Likelihood (ML) estimatesof the parameters of the distribution in the presence of missing or hidden data. The name EM algorithm was given by A. P. Dempster; N. M. Laird; D. B. Rubin in their fundamental paper. We apply the EM algorithm to find the MLEs of the parameters of the distribution. Eachiteration of the EM algorithm consists of two steps:

- The Expectation or E-step, and

- The Maximization or M-step.

In the expectation, or E-step, the missing data are estimated given the observed data and current estimate of the model parameters. This is achieved using the conditional expectation, explaining the choice of terminology.

In the M-step, the likelihood function is maximized under the assumption that the missing data are known. 


\section{Analysis of Example Data Set}

In this paper we analyze a set of failure data of a particular type of battery that used in automobile and trailer. The data set is an incomplete failure data on a sample of 54 batteries. The data include failure times for 39 items that failed under warranty and service times (censored times) for 15 items that had not failed at the time of observation. The measure unit of the lifetime is given in Days. This data set is given in Blischke, Karim and Murthy (2011).

\subsection{Model Selection for the Data}

Blischke et al. (2011) analyzed this battery failure data assuming a 2-parameter Weibull distributionas a reasonable choice for the data. They found the maximum likelihood estimates of the shape parameter $\hat{\beta}=1.9662$ and scale parameter $\hat{\eta}=836.344$.

Using information from the service center (maintenance department) it can be assumed that there are two types of customers. One type of customers maintain the battery regularly and properly (say correct maintenance) and the other type of customers did not do maintenance properly (say incorrect maintenance). The basic tasks related to battery maintenance are: keeping the water level at the fill line; cleaning any corrosion off the battery terminals; and checking and keeping the batteries charge correctly. Since the failure data may have come from two sub-populations, we can assume a mixture model for the data. Therefore, we assume a two-fold Weibull mixture model instead of a single Weibull model. The CDF of this model becomes

$$
\begin{aligned}
& F(t)=p F_{1}(t)+(1-p) F_{2}(t) \\
& \quad=\left[p\left\{1-\exp \left[-\left(\frac{t}{\eta_{1}}\right)^{\beta_{1}}\right]\right\}+(1-p)\left\{1-\exp \left[-\left(\frac{t}{\eta_{2}}\right)^{\beta_{2}}\right]\right\}\right], \mathrm{t} \geq 0
\end{aligned}
$$

Table 1. MLEs of the Parameters of Weibull-Weibull mixture model.

\begin{tabular}{cl}
\hline PARAMETER & MLE (VIA EM ALGORITHM) \\
\hline$\beta_{1}$ & 4.0446598 \\
$\beta_{2}$ & 1.3559817 \\
$\eta_{1}$ & 801.1941526 \\
$\eta_{2}$ & 880.8844635 \\
$p_{1}=p$ & 0.4754753 \\
$p_{2}=1-p$ & 0.5245247 \\
\hline
\end{tabular}

For estimating the five parameters $\left\{\beta_{1}, \eta_{1}, \beta_{2}, \eta_{2}, p\right\}$ of the 2-fold Weibull mixture model, the EM algorithm is applied. The maximum likelihood estimates of the parameters are given in Table 1. The mean for a Weibull model with shape parameter $\beta$ and scale parameter $\eta$ is given by $\eta \Gamma(1+1 / \beta)$ where $\Gamma(\cdot)$ is the gamma function. For the 2-fold Weibull mixture model, the mean for $F_{2}(t)=$ 807.13 days $>$ mean for $F_{1}(t)=726.66$ days. Therefore, it can be concluded that in the mixture model $F_{1}(t)$ be the distribution for the incorrect maintenance batteries and $F_{2}(t)$ be the distribution for the correct maintenance batteries. And the estimates of $p_{1}$ and $p_{2}$ indicate that there are $48 \%$ incorrect maintenance batteries and $52 \%$ correct maintenance batteries.

To check the fit, we find the nonparametric estimate of the CDF based on the Kaplan-Meier (KM) estimation method. Also we find the ML estimates of the CDFs of two competitive models - single Weibull model and 2-fold Weibull mixture model. The plots are shown in Fig. 2. The figure indicates that theCDF of mixture model is much closer to nonparametric CDF than the CDF of single Weibull model. Therefore, the 2-fold Weibull mixture model can be selected for the data.

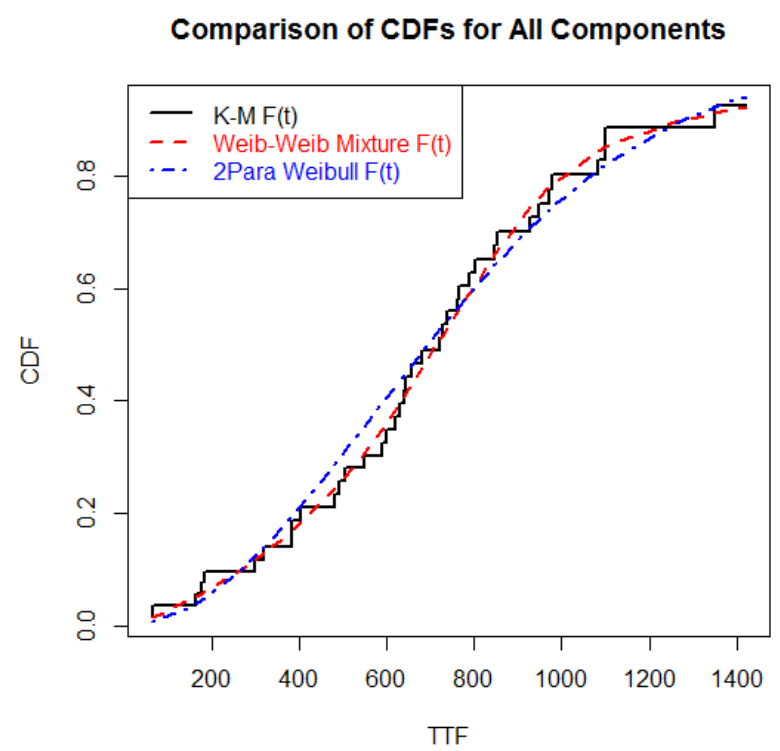

Figure 2. Comparison of Single and 2-fold WeibullmixtureCDFs with empirical $C D F$

The predicted reliabilitiesof battery up to 2500 days based on single Weibullmodel and 2-foldWeibullmixture model are shown in Fig.3. This plot also shows Kaplan-Meier estimate for given maximum lifetimes up to 1421 days.

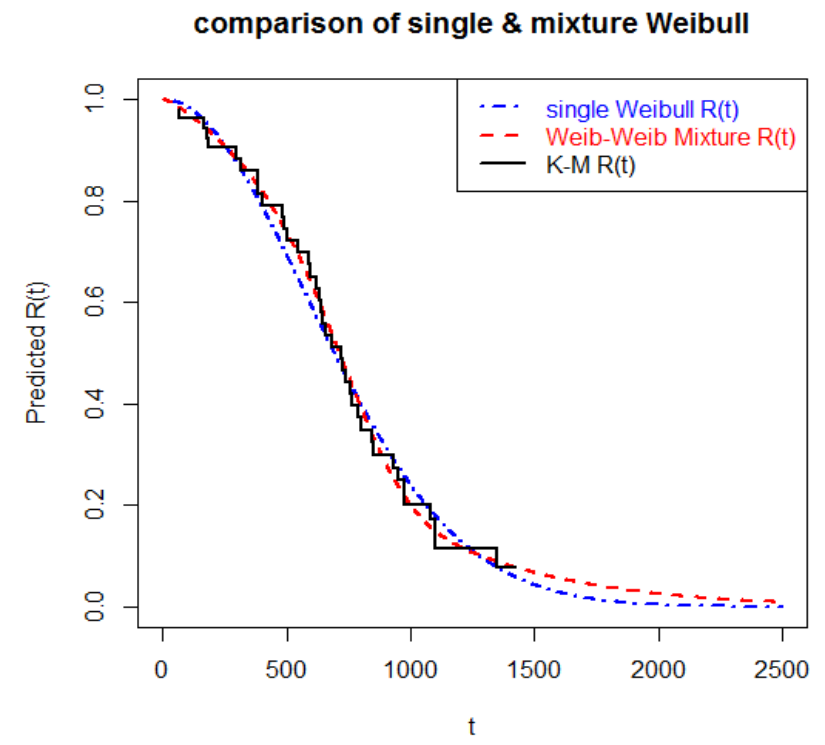

Figure 3. Plots of predicted reliability function $R(t)$ 
Fig. 3 indicates that the mixture model is more compatible than the single Weibull model. Thus in the next Section we use the 2-fold Weibull mixture model for estimating the optimum maintenance age for the battery.

A comparative study of the estimates of reliability functions for non-parametric, single Weibull and WeibullWeibull mixture models are given in Table2.

Table 2. Comparison of the Estimates of Reliability Functions

\begin{tabular}{llll}
\hline T & $\begin{array}{l}\text { K-M ESTIMATE } \\
\text { OF CDF }\end{array}$ & $\begin{array}{l}\text { SINGLE } \\
\text { WEIBULL R(T) }\end{array}$ & $\begin{array}{l}\text { MIXTURE } \\
\text { WEIBULL R(T) }\end{array}$ \\
\hline 506 & 0.72041 & 0.68913 & 0.73417 \\
983 & 0.19919 & 0.25310 & 0.21267 \\
1421 & 0.07588 & 0.05863 & 0.07749 \\
1750 & - & 0.01397 & 0.04151 \\
2000 & - & 0.00387 & 0.02508 \\
2200 & - & 0.00123 & 0.01649 \\
2500 & - & 0.00018 & 0.00856 \\
\hline
\end{tabular}

The single Weibull model estimate the probability that the system (battery) survives up to 506 days is 0.69 whereas the mixture model estimate this as 0.73 and $\mathrm{K}-\mathrm{M}$ estimate this0.72, which indicate mixture model is close to the nonparametric estimate and Weibull model under estimate the reliability than that of mixture model.

Single Weibull model suggest that there is $25 \%$ chance that the system will survive up to 983 days, at the same time mixture model estimates this chance as $21 \%$ that is close to K-M estimate i.e. $20 \%$.

Again,single Weibull model estimates the reliability for 1421 days is 5\% whereas $\mathrm{K}-\mathrm{M}$ estimate gives $7 \%$ and mixture Weibullmodel also shows $7 \%$ as well.

Themaximum likelihood estimates of MTTF (Mean Time to Failure) for single Weibull and mixture Weibull modelsare given in Table3.

Table 3. MTTF for single Weibull and mixture Weibull models

\begin{tabular}{ll}
\hline MTTF \\
\hline Single Weibull & Mixture Weibull \\
\hline 741.4486 & 768.8706 \\
\hline
\end{tabular}

Table 3shows that the single Weibull model under estimate the MTTF than the mixture Weibull model.

\subsection{Optimal Replacement Age}

Since the 2-fold Weibull mixture model fits the data well, this model will be used for finding out the optimal age based on the minimum maintenance cost.

This objective function for the maintenance cost needs preventive and corrective maintenance costs denoted by $C_{p}$ and $C_{f}$ respectively. Both of these costs involve some kinds of direct and indirect expenses. But here these expenses are not given separately for this data set.

Fig. 4shows the objective function $J(T)$ with respect to time, $T$, for the assumed values of $C_{p}=\$ 50$ and $C_{f}=\$ 500$.

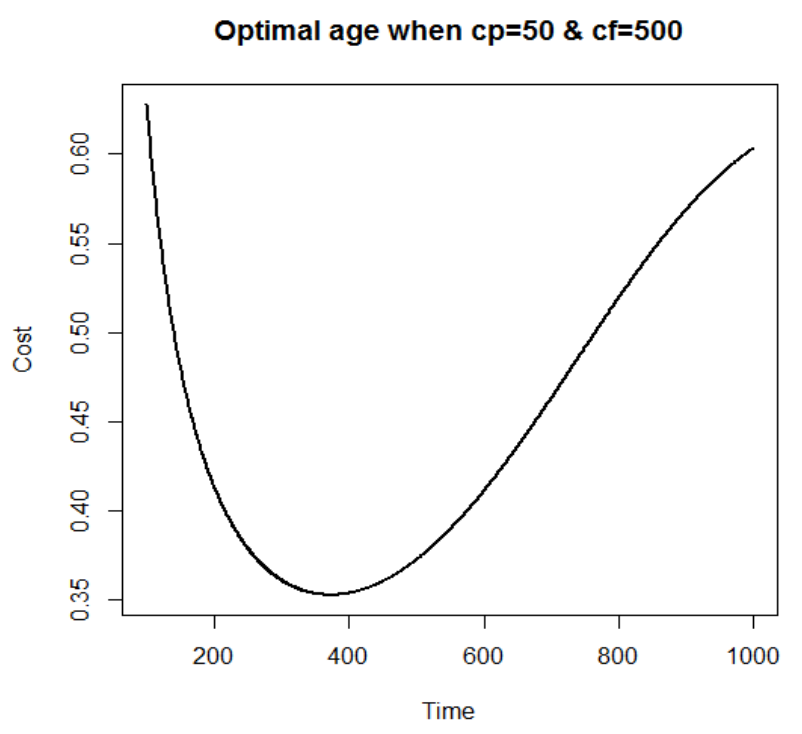

Figure 4. Optimal Maintenance Age for $C_{p}=50$ and $C_{f}=500$

Fig. 4shows the optimal age $T^{*}=372$ days that minimizes the expected cost rate function at the value of $J\left(T^{*}\right)=\$ 0.353$. This suggests that the optimum maintenance age is 372 days for $C_{p}=50$ and $C_{f}=500$.

The optimum maintenance ages and costs for a variety choices of $C_{p}$ and $C_{f}$ are given in Table 4 . This table can be useful to the manufacturer or owner for selecting the optimum maintenance age of the Battery if the preventive and corrective maintenance costs vary.

Table 4. Optimal age and cost for different values of $C_{p}$ and $C_{f}$

\begin{tabular}{cllllllllllll}
\hline $\boldsymbol{C}_{\boldsymbol{f}}$ & 300 & 300 & 300 & 300 & 400 & 400 & 400 & 400 & 500 & 500 & 500 & 500 \\
$\boldsymbol{C}_{\boldsymbol{p}}$ & 10 & 15 & 20 & 25 & 10 & 15 & 20 & 25 & 25 & 50 & 75 & 100 \\
$\boldsymbol{T}^{*}$ & 230 & 281 & 318 & 347 & 196 & 245 & 281 & 309 & 281 & 372 & 431 & 479 \\
$\boldsymbol{J}\left(\boldsymbol{T}^{*}\right)$ & 0.152 & 0.169 & 0.186 & 0.199 & 0.187 & 0.208 & 0.226 & 0.243 & 0.283 & 0.353 & 0.407 & .456 \\
\hline
\end{tabular}

\section{Conclusion}

To find the optimal maintenance age at which the nonfailed battery to be maintained, first a 2-fold Weibull mixture model is selected as the best model for the lifetime of the battery. This paper identified that in the 2-fold mixture model,one be the distribution for the incorrect maintenance batteries and another be the distribution for the correctly maintenance batteries. The estimates indicated that there are $48 \%$ incorrect maintenance batteries and $52 \%$ 
correctlymaintenance batteries. Based on this analysis, we decided the optimal maintenance age is 372 days because that age has the lowest cost in maintenance purposes i.e. $\$ 0.353$.The main contribution of this research is to find the optimal maintenance age at which the non-failed battery be maintained. This research should be of interest and useful to maintenance professionals, researchers, practitioners, those aspiring to be maintenance managers and persons with quality, production and related areas.

\section{References}

[1] Aditya, P. and Uday, K., 2006. Maintenance performance measurement (MPM): issues and challenges. Journal of Quality in Maintenance Engineering, 12(3), pp.239-251.

[2] Blischke, W.R., Karim, M.R. and Murthy, D.N.P. (2011), Warranty Data Collection and Analysis, Springer Verlag, London.
[3] Duffuaa,S.O., Raouf,A.andCambell,J.D. (1999), planning and control of maintenance systems:modeling and analysis, John Wiley \& sons.

[4] Eichler, C. (1990). Instandhaltungstechniken. VerlagTechnik $\mathrm{GmbH}$, Berlin.

[5] Hale AH, Heming BHJ, Smit K, Rodenburg FG, Van Leeuwen ND (1998) Evaluating Safety in the Management of Maintenance Activities in the Process Industry. SafSci28(1): $21-44$.

[6] Mobley, R. K., 2004. Maintenance fundamentals. 2nd ed. Burlington, Mass.: Butterworth Heinemann.

[7] U. Kumar, D. Galar, A. Parida, C.Stenström, L. Berges, Maintenance Performance Metrics: A State of the Art Review, ISBN 978-91-7439-379-8. 